\title{
Modélisation Du Cycle Des Roches Sédimentaires Au Profit Des Apprenants De La Première Année Collégiale
}

\author{
Amina Tajdi \\ Ali Nazih \\ Atika Eddif
}

Equipe de recherche: Recherche en Science et en Didactique, Centre Régional des Métiers de l'Education et de la Formation Fès- Meknès, Maroc

Jamila Kenafi

Centre Régional des Métiers de l’Education

et de la Formation De l’Oriental, Hay al massira; Oujda, Maroc

\section{Issam Mahboubi \\ Jihad Oubezza \\ Et Tarik Chelouati}

Equipe de recherche: Recherche en Science et en Didactique, Centre Régional des Métiers de l'Education et de la Formation Fès- Meknès, Maroc

doi: 10.19044/esj.2017.v13n16p146 URL:http://dx.doi.org/10.19044/esj.2017.v13n16p146

\begin{abstract}
The present study aims to identify the difficulties to assimilate the concept of the sedimentary rocks cycle for first year middle high school students. It also aims to test the importance of a physical model that we have proposed. In order to collect the didactic difficulties encountered in the classroom as well as the means suggested to overcome them, we administered an anonymous questionnaire to 44 teachers of Life and Earth Sciences. To test the significance of the model, 184 learners were divided into two groups. While the first group was taught using a model, no specific one was used to teach the other group. After a constructive evaluation, 73\% of the learners in the first group achieved satisfactory results, against 34\% in the second one.
\end{abstract}

Keywords: Sedimentary rocks cycle, model, assimilation, learners

\section{Résumé}

La présente étude se propose d'identifier les difficultés à assimiler le concept du cycle des roches sédimentaires en 1ère année collège et 
d’éprouver l'intérêt d'un modèle physique que nous avons proposé. Pour recueillir les difficultés didactiques rencontrées en classe ainsi que les moyens proposés pour les surmonter, nous avons recours à un questionnaire anonyme distribué à 44 enseignants des Sciences de la Vie et de la Terre. Pour éprouver l’intérêt du modèle 184 apprenants ont été répartis en deux groupes; le premier a été enseigné avec modèle et l'autre sans modèle. Après évaluation constructive, $73 \%$ des apprenants du 1er groupe ont réalisé des résultats satisfaisants, contre 34\% dans le 2ème groupe.

Mots clés: Cycle des roches sédimentaires, modèle, assimilation, apprenants

\section{Introduction}

Les sciences de la Terre sont des sciences pluridisciplinaires qui intègrent des connaissances spécialisées à d’autres disciplines, essentiellement les mathématiques, la physique et la chimie. Les apprenants doivent alors avoir des pré requis suffisants en ces matières. En outre, les notions du temps et de l'espace sur lesquelles la discipline est basée, portent sur une échelle qui dépasse l'expérience individuelle des apprenants. Ces derniers trouvent des difficultés à imaginer des phénomènes s’étalant sur des millions et des milliards d'années, malgré le fait de leur montrer clairement les indices et les résultats de ces phénomènes dans la nature. De même, ils ont des difficultés à passer du plan (2D) au volume (3D) et inversement.

Ces caractéristiques propres aux sciences de la Terre, ont contribué à la dévalorisation de cette discipline auprès du public visé, à savoir les apprenants et même dans les programmes scolaires, conformément aux travaux de Gould (1990), de A. Bezzi (1999), de G. Gohau (2001) \&, J. Dodick et N. Orion (2003). Cela a amené des spécialistes à s’interroger sur la manière dont doit être conçu leur enseignement. Ainsi, Ces dernières années, plusieurs travaux montrent l'importance des modèles et de la modélisation dans l'enseignement des sciences de la terre, notamment ceux de Halwachs (1975), Bachelard (1979); Drouin (1988); Martinand (1992); Orange (1997) ; Clement (2000) ; B.-C. Bukley, (2000) et Orange (2003). En accord avec ces travaux, notre étude s'est fixée comme objectif de proposer une technique pour modéliser le processus géologique appelé le Cycles des Roches Sédimentaires (CRS) et d'évaluer son impact sur la qualité des apprentissages. Ce concept fait partie du programme de la première année de l’enseignement collégial marocain.

\section{Matériel et méthodes}

Notre étude est basée sur un questionnaire distribué à 44 enseignants des Sciences de la Vie et de la Terre au collège, et un test d'évaluation sur 
184 apprenants en première année de collège répartis en 4 classes à l'établissement Bnou Otmane El Meknassi, délégation de Meknès.

\section{Le Questionnaire}

Notre questionnaire comporte une dizaine de questions destinées à 44 enseignants de collège des SVT, à l'échelle de la délégation de Meknès. Nous leurs avons demandé essentiellement :

- Si les difficultés d'assimilation du concept sont liées à l'âge (maturité neuro-intellectuelle des apprenants), aux moyens didactiques et pédagogiques, à la complexité du concept en lui-même, ou à d'autres facteurs?

- Les solutions qu'ils proposent pour remédier au problème ?

Après le dépouillement, les résultats ont été représentés sous forme de graphes.

\section{Le Modèle proposé}

Le modèle proposé est constitué de :

- Un support en bois : pour le maintien de la topographie du bassin. Il est revêtu par une plaque en plastique flexible et imperméable pour protéger le bois.

- Un relief : construit par du plâtre et épousant la topographie, depuis les «hautes montagnes» en amont jusqu'à «la mer» en aval. Pour l'apparence, le relief est ensuite teinté de vert (végétation) et de jaune ocre (sols).

- Un aquarium : il est en verre et permet le regroupement des différents constituants. Il est de $80 \mathrm{~cm}$ de longueur, $30 \mathrm{~cm}$ de largeur et 40 cm de hauteur (Voir photos 1).

L'édifice final a l'avantage d'être facile à transporter puisqu'il est démontable en deux pièces (le support en bois et l'aquarium en verre) et il ne pèse qu'environ $12 \mathrm{~kg}$.

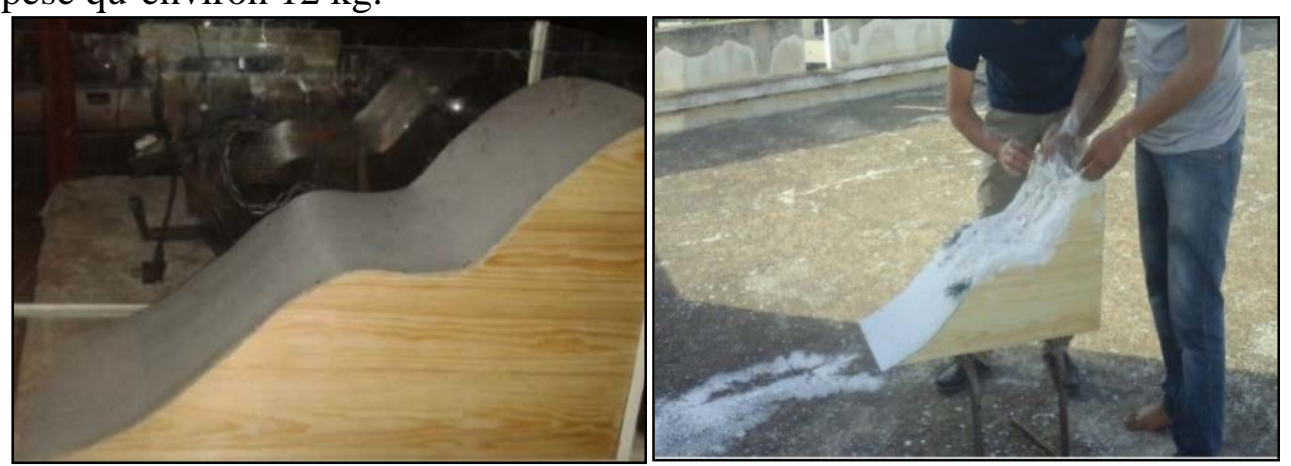

Photo 1 : modèle en réalisation 


\section{Exploitation du modèle}

Quatre classes de la première année du collège, soit 184 élèves âgés entre 11 et 14 ans, ont participé à cette étude. Ils n’avaient pas des pré requis sur la notion du CRS durant tout leur cursus scolaire. Ils ont été systématiquement répartis en deux groupes de deux classes chacun. Le premier a été enseigné avec modèle et le deuxième sans modèle mais avec d'autres supports didactiques, essentiellement, des schémas explicatifs au tableau, des photos et les schémas du manuel scolaire « $\mathrm{Al}$ wadih ».

\section{Présentation du modèle} modèle

Nous avons demandé aux élèves de faire une description de ce

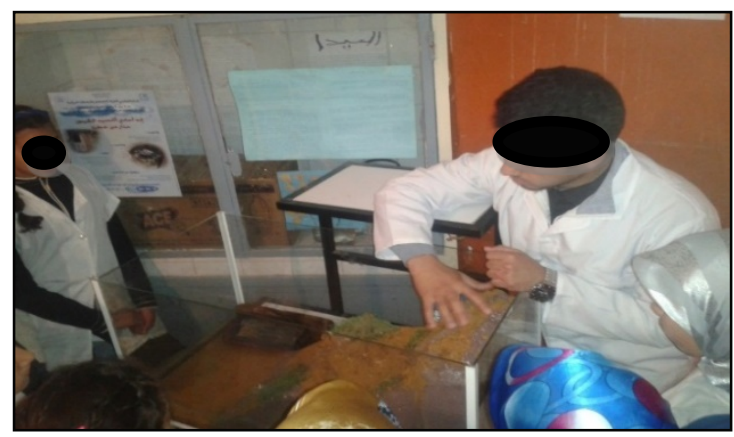

Photo 2 : le modèle réalisé

\section{Mise en ouvre du modèle}

En amont les apprenants ont posé des particules de tailles différentes, et ils ont simulé les précipitations par arrosage (photo 3), en même temps, le professeur les oriente à suivre le devenir des particules.

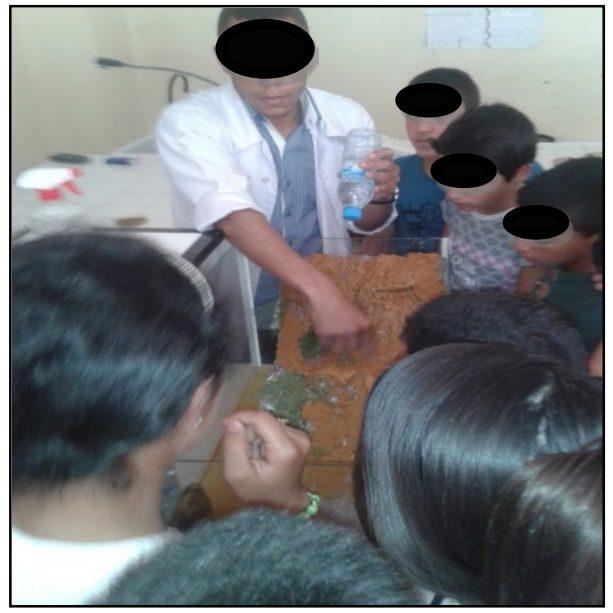

Photo 3 : simulation des précipitations 


\section{l'Evaluation constructive}

L'évaluation constructive est sous-forme d'une question claire et simple. Nous avions demandé aux apprenants de reproduire les principales étapes du cycle des roches sédimentaires.

\section{Résultats et Discussion}

\section{Les résultats de l'investigation par questionnaire}

$80 \%$ des enseignants ayant rempli le questionnaire avaient au moins 20 ans d'expérience dans l'enseignement, dont 15ans en moyenne dans l'enseignement de la première année de collège.

\section{Existence des difficultés d'assimilation du concept}

La plupart des enseignants ont confirmé l'existence d'énormes difficultés à aborder cette notion pour la première fois avec leurs élèves. 80 $\%$ ont déclaré que, malgré leurs efforts, les résultats sont souvent décevants chez leurs apprenants. Ils ont également confirmé que la plupart de leurs élèves n'arrivent pas à assimiler les notions du temps, d'espace et de la cyclicité des événements. En accord avec les travaux Gould (1990), Ault (1994), Savaton, (1998), Trend (2000) et Raab \& Frodeman (2002).

\section{Les origines des difficultés d'assimilation :}

Les origines des difficultés d'assimilation du concept, selon les enseignants interrogés, sont présentées dans la figure 1.

Figure 1 : origines des difficultés d'assimilation du CRS

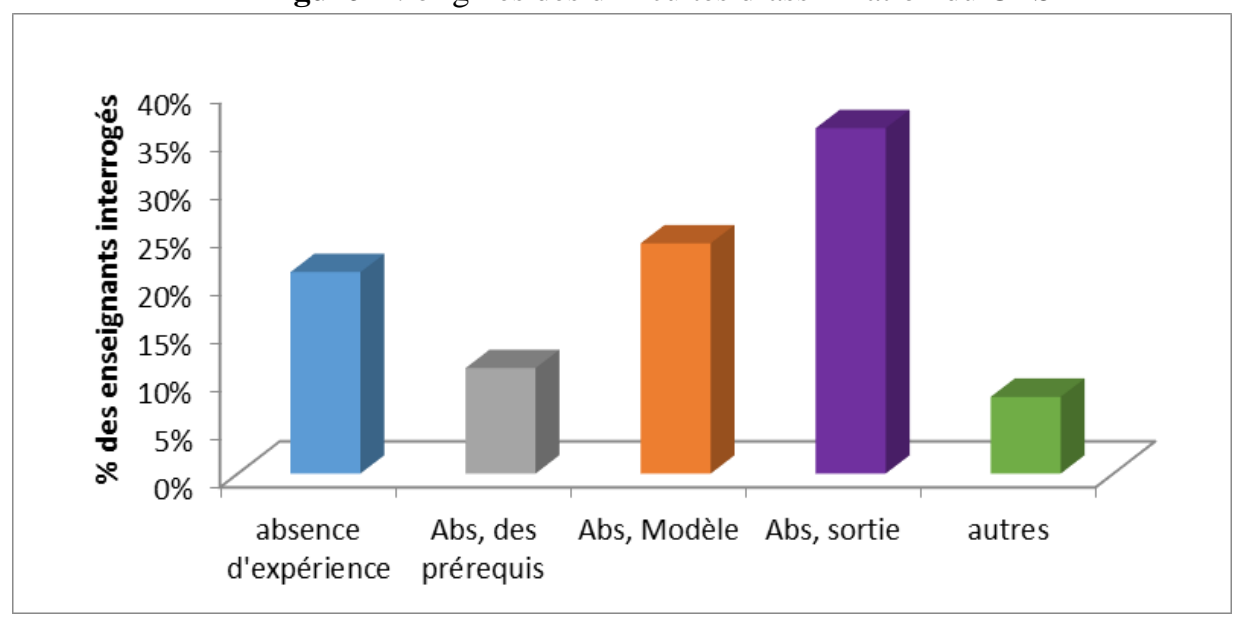

36\% des enseignants interrogés imputent ces difficultés à l'absence de la sortie au terrain, $24 \%$ à l'absence d'un modèle explicatif,21\% à l'absence des expériences du laboratoire ; $11 \%$ aux pré requis des élèves et $8 \%$ à d'autres facteurs. 


\section{Insuffisance du temps d'apprentissage}

Plus de $50 \%$ des enseignants interrogés indiquent que le volume horaire réservé à la notion du C.R.S dans la 1ere année du collège est insuffisant pour que les élèves puissent mieux assimiler le concept.

\section{Les méthode proposées pour la construction des concepts du C.R.S}

Concernant l'efficacité des méthodes de construction des concepts du C.R.S : $44,6 \%$ trouvent que la sortie au terrain est la plus convenable, $39.8 \%$ ont choisi la modélisation comme meilleure méthode à adopter et $15,7 \%$ des enseignants préfèrent l'expérimentation au laboratoire.

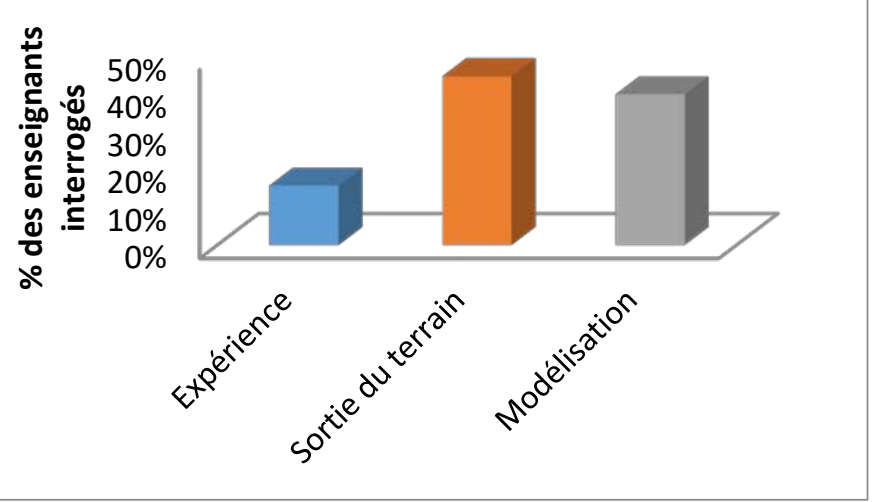

Figure 2 : la méthode idéale pour la construction des concepts du C.R. S

\section{Les moyens effectivement utilisés :}

Même si la majorité des enseignants interrogés insistent sur l'importance de la sortie géologique dans la construction du savoir en géologie, seulement $2 \%$ d'entre eux arrivent à la réaliser.

Pour remédier à l'absence de la sortie au terrain : 48,1\% ont recours à la modélisation ;24,7\% réalisent des manipulations du laboratoire ; 14,8\% préconisent le travail par groupe et $12,3 \%$ des enseignants préfèrent les schémas. (Figure 3)

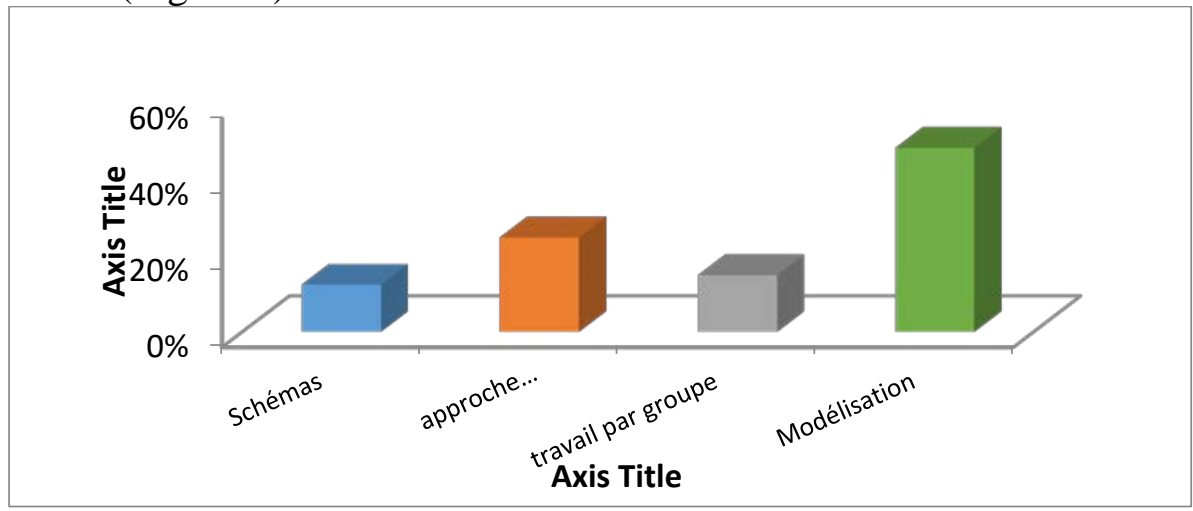

Figure 3: Meilleure méthode pour expliquer le C.R.S 
Il est à souligner que les enseignants ayant mentionné avoir recours à la modélisation de C.R.S, ne réalisent que des manipulations pour chaque étape de ce cycle, $21,1 \%$ des enseignants les utilisent pour expliquer l'érosion, 28,9\% les utilisent pour expliquer le transport, 36,7\% pour la sédimentation et $13.3 \%$ pour la diagenèse.

Concernant la possibilité que les TICE peuvent remplacer l'utilisation des modèles, 93\% des enseignants disent que c'est possible, par contre, 7\% voient que les TICE ne peuvent pas remplacer la modélisation.

\section{Exploitation du modèle}

Erosion mécanique à l'amont ; Transport des particules érodées le long des canaux de ruissellement ; Sédimentation de ces particules au niveau des bassins de sédimentation (voir photo 4).

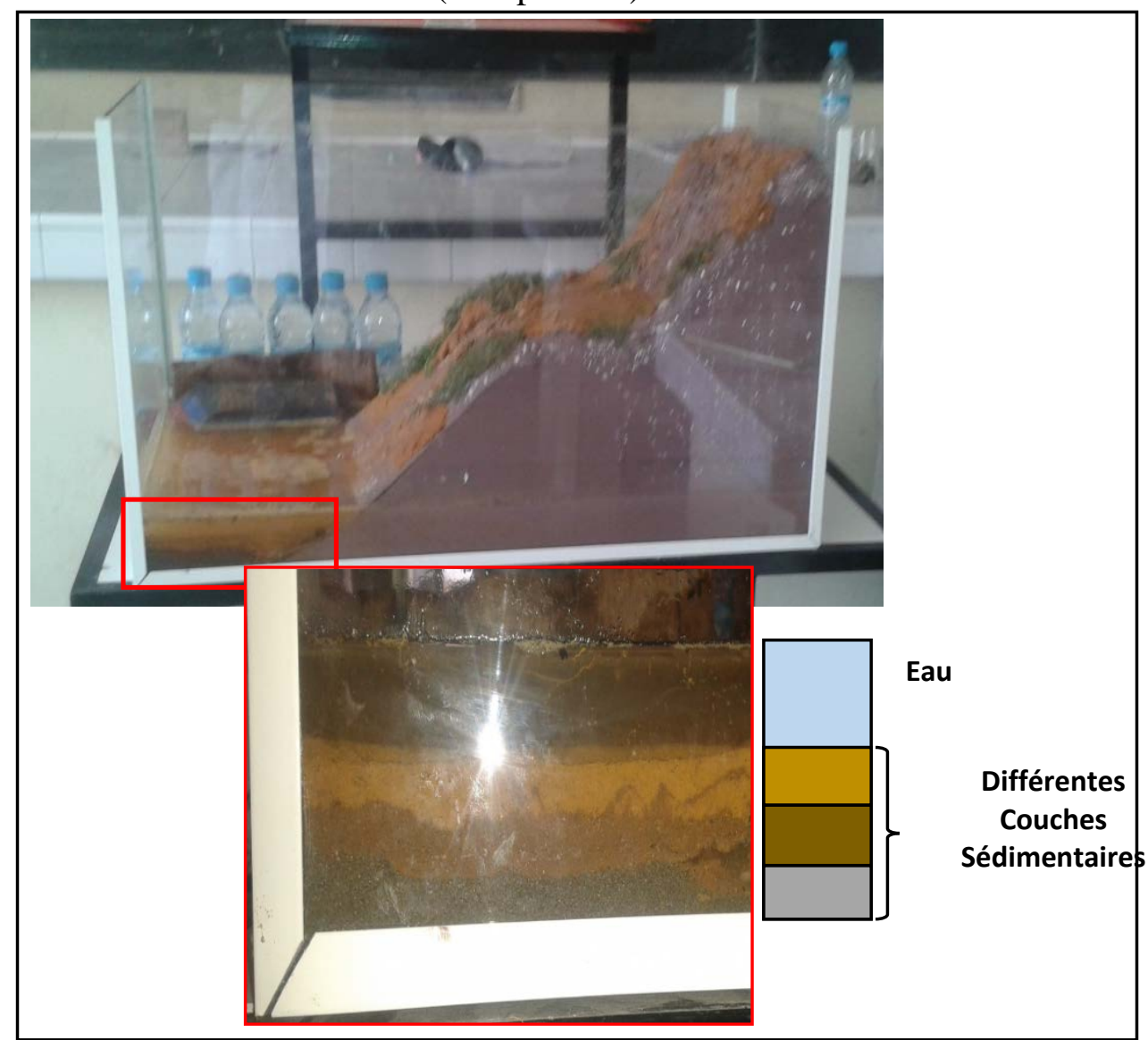

Photo 4 : la sédimentation

La simulation de la diagénèse est réalisée à l'aide des manipulations additives au modèle. 
Pour mettre en évidence la mise à l'affleurement, les pré-requis des apprenants lors de la sortie géologique étaient nécessaires. Les apprenants ont résumé les différentes étapes du CRS sous-forme d'un schéma récapitulatif semblable à la forme du modèle réalisé.

\section{Evaluation constructive}

Pour ce faire, nous avons recours à deux groupes d'apprenants en première année du collège. Le premier groupe avait étudié le C.R.S sans modèle. Le deuxième groupe a bénéficié du modèle.

Nous avons ensuite effectué le même test d'évaluation pour les deux groupes.

\section{Le groupe sans modèle}

$34 \%$ seulement ont montré qu'ils ont assimilé le concept. Alors que 66\% ont donné une mauvaise réponse.

Pour ces apprenants testés sur le C.R.S sans modélisation, nous présentons ci-dessous des exemples de réponses considérées acceptables ou non acceptables.

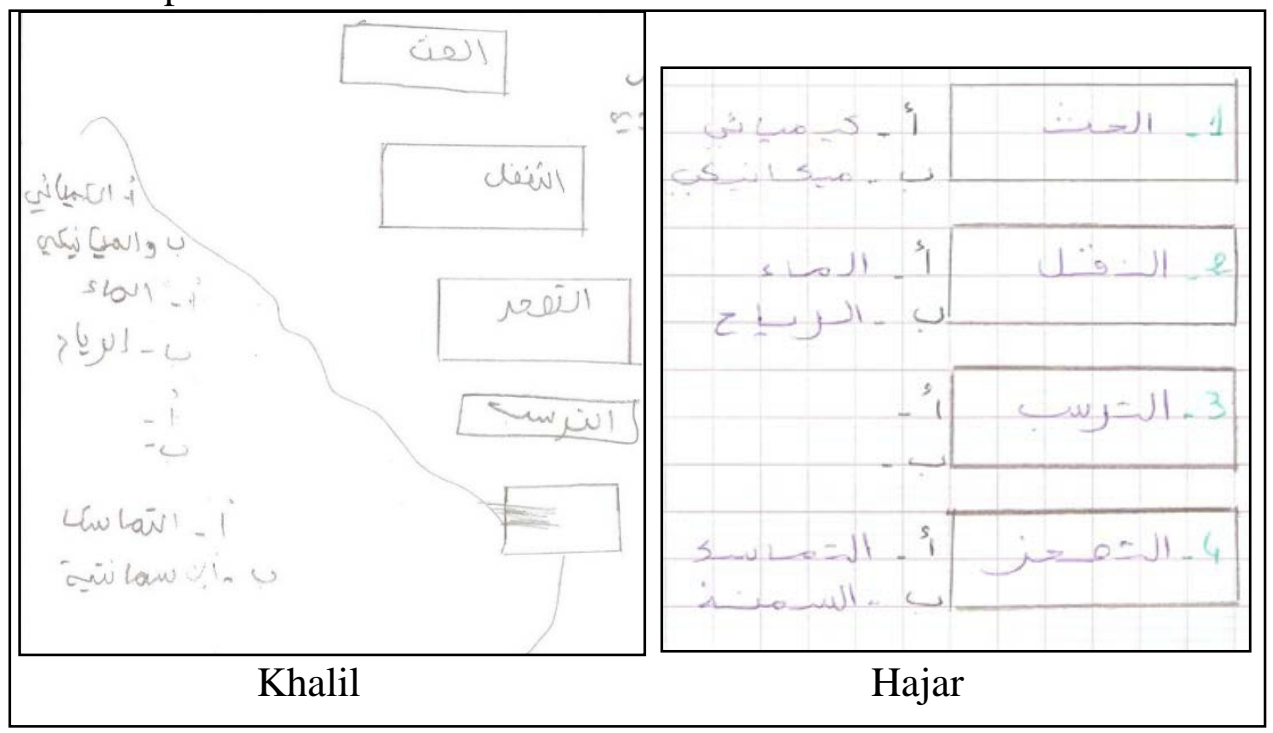

Photos 5 : Exemples des copies des apprenants (Le groupe sans modèle).

\section{Le groupe avec modèle}

$73 \%$ ont des apprenants ont présenté des réponses admises, alors que seulement $27 \%$ n’ont pas bien assimilé le concept.

Nous exposons ci-dessous des exemples de réponses considérées acceptables ou non acceptables. 


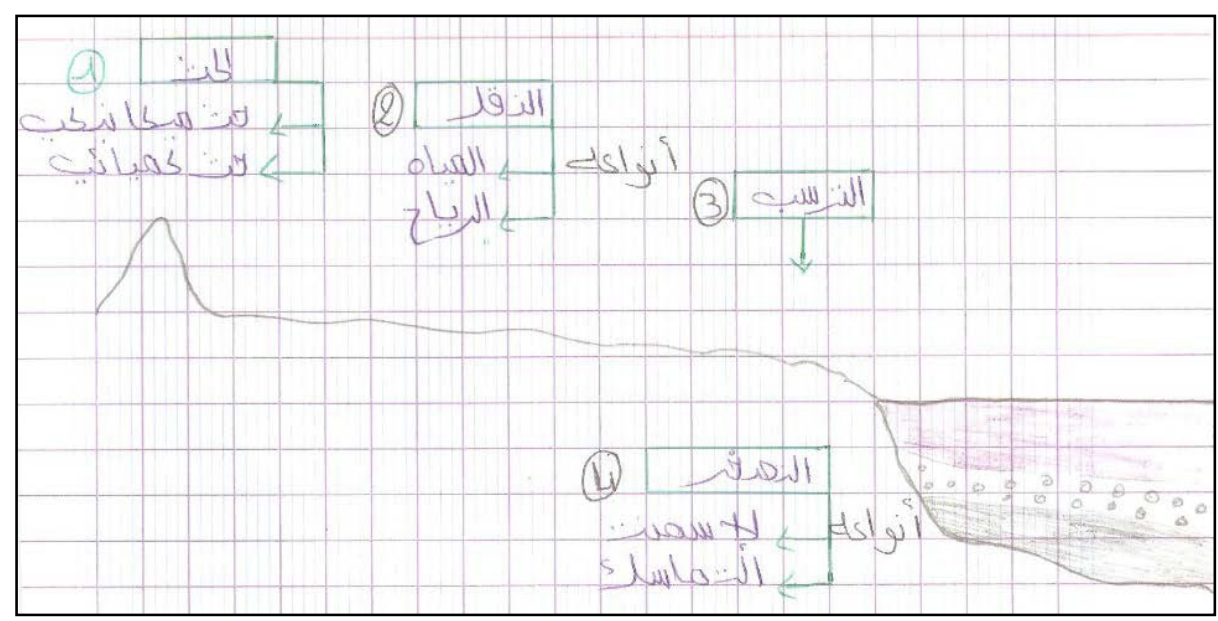

Salma

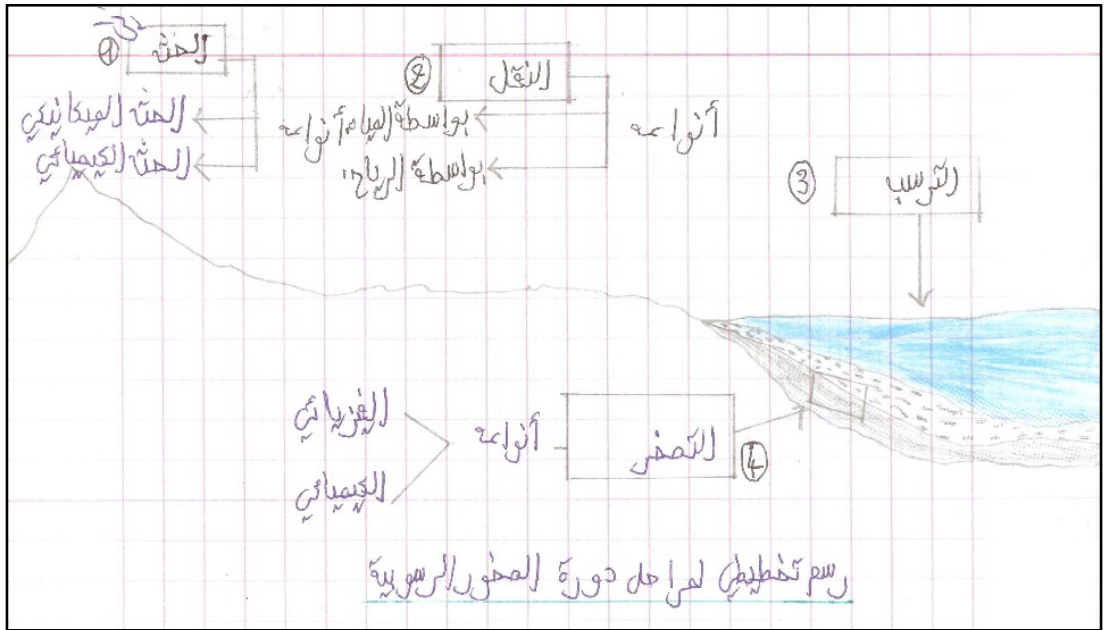

Youssef

Photos 6: Exemples des copies des apprenants (Le groupe avec modèle).

\section{Conclusion}

Cette étude a montré que notre modèle physique est un outil didactique qui a facilité l'assimilation d'un concept géologique très complexe (le cycle des roches sédimentaires), chez une catégorie d'âge des apprenants moins avertie (les collégiens). Les apprenants ayant bénéficié du modèle, ont bien situé les différentes étapes du processus dans le temps et dans l'espace. D'ailleurs, c'est effectivement l'une des principales difficultés rencontrées en géologie, car ce genre de phénomène naturel dépasse l'expérience individuelle des enfants.

Cependant, il y'a lieu de noter que notre modèle est encore loin d'être performant. Nous espérons ainsi:

- compléter techniquement l'étape de la diagenèse ; 
- simuler les conditions de mise en affleurement des roches sédimentaires formées,

- retravailler l'échelle de chaque constituant dans le modèle ; et ce, afin de pouvoir le généraliser dans d’autres établissements scolaires.

\section{References:}

1. AULT C. R. (1994). Research on problem solving: Earth science. In D. L. Gabel. Handbook of research on science teaching and learning. Macmillan Publishing Company.

2. BACHELARD S. (1979). Quelques aspects historiques des notions de modèle et de justification des modèles. In $\mathrm{P}$. Delattre et $\mathrm{M}$. Thellier. Elaboration et justification des modèles. Maloine.

3. BEZZI A. (1999). what is thing called geoscience. Epistemological dimension elicited with the repertory grid and their implications for scientific literacy. Science Education vol 83, Issue 6, 675-700

4. BUKLEY, B.-C. (2000). Interactive and model-based learning in biology. International Journal of Science Education (22), 895-935.

5. CLEMENT, J. (2000). Model based learning as a key research area for science education. International Journal of Science Education (22), 1041 -1053.

6. DROUIN AM. (1988). «Le modèle en question » Aster 7, pp 1 à 20, Paris, INRP.

7. DODICK, J. \& ORION, N. (2003). "Geology as an historical science: Its perception within science and the educationsystem". Science \& Education, 12, 197-211.

8. GOHAU G., (2001). La géologie, discipline mal aimée, dans Etudes sur l'enseignement des sciences physiques e tnaturelles, Cahiers d'histoire et de philosophie des sciences, textes réunis par Nicole Hulin, ENS éditions.

9. GOULD, S-J.(1990) . Aux racines du temps, Grasset et Fasquelle.

10. HALBWACHS F. (1975). «La physique du maître entre la physique du physicien et la physique de l'élève » Revue française de pédagogie 33, INRP.

11. MARTINAND JL. (1992). Problèmes et modélisation en biologie, quels apprentissages pour le lycée ? Paris : PUF.

12. ORANGE C. (1997). Problèmes et modélisation en biologie, Paris, PUF

13. ORANGE D. (2003b). Utilisation du temps et explications en sciences de la Terre par les élèves de lycée : étude dans quelques problèmes géologiques. Thèse de doctorat de l'Université de Nantes, Nantes. 
14. RAAB, T., \& FRODEMAN, R. (2002). What's it like to be a geologist? Phenomenology of geology and its practical implications. Philosophy an Geography, 5 (1), 69-81.

15. SAVATON P. (1998). L'enseignement de la carte géologique dans le secondaire : Bilan historique et didactique; réflexion et propositions d'apprentissage nouveau. Thèse Paris 7

16. TREND R. (2000). “Conceptions of geological time among primary teacher trainees, with reference to their engagement with geoscience, history, and science". International Journal of Sciences Education, vol 22. 